\title{
Total Knee Arthroplasty in a Case of Tuberculosis Knee in Healing Stage: Is it Safe?
}

\author{
${ }^{1}$ Sanjay Yadav, ${ }^{2}$ Chandra Shekhar Yadav, ${ }^{3}$ Nishikant Kumar, ${ }^{4}$ Ashok Kumar
}

\section{ABSTRACT}

Background: Association of tuberculosis and total hip joint replacement (THR) is described with or without antitubercular treatment (ATT) cover but total knee arthroplasty (TKA) in early disease in single-stage is uncommonly reported. We wish to share our clinical experience in such a case.

Case description: A 22-year-old male presented with pain and swelling of left knee for 7 months. It was drained at local health facility with sinus development. Tuberculosis was diagnosed by clinic-radiological evaluation and ATT was started. Sinus healed but disabling pain and knee stiffness with flexion deformity persisted. Radiographs revealed destructive osteoarticular arthritis with periarticular osteopenia. Debridement followed by TKA in single-stage was done with posterior-stabilized implant. Postoperative period was uneventful. Histopathological analysis confirmed chronic granulomatous synovitis with caseation necrosis suggestive of mycobacterial infection. After 1-year, ATT was discontinued and patient was disease-free.

Clinical relevance: Single-stage prosthetic knee joint arthroplasty can be safely performed under adequate ATT coverage in early disease provided response to medical treatment is good. Patient counseling regarding compliance with ATT and postoperative protocol is important in ensuring success.

Keywords: Tuberculosis, Total knee arthroplasty, TKA.

How to cite this article: Yadav S, Yadav CS, Kumar N, Kumar A. Total Knee Arthroplasty in a Case of Tuberculosis Knee in Healing Stage: Is it Safe? J Postgrad Med Edu Res 2015;49(3): 139-142.

\section{Source of support: Nil}

Conflict of interest: None

\section{INTRODUCTION}

Osteoarticular tuberculosis remains one of the common forms of extrapulmonary forms of tuberculosis and among these, tuberculosis of the knee joint ranks after spinal and hip tuberculosis. ${ }^{1,2}$ Since, majority of the

\footnotetext{
${ }^{1}$ Senior Resident, ${ }^{2}$ Professor, ${ }^{3}$ Senior Research Officer ${ }^{4}$ Assistant Professor

${ }^{1-4}$ Department of Orthopedics, All India Institute of Medical Sciences, New Delhi, India
}

Corresponding Author: Sanjay Yadav, Senior Resident Department of Orthopedics, All India Institute of Medical Sciences, New Delhi, India, Phone: 9560754591, e-mail: drsanjay.pgi@gmail.com individuals affected with osteoarticular tuberculosis fall between 20 and 40 years of age which being the most productive age group, leads to significant psychosocial and financial issues. Chemotherapy constitutes the cornerstone of treatment with surgery playing an adjunctive role mainly in cases with inadequate response. ${ }^{3}$ However, treatment is prolonged and in view of the often delayed presentation, functional recovery remains incomplete.

Recently, there have been several reports of successful management of tuberculous arthritis of the knee with primary total knee arthroplasty (TKA) with knee replacement often being done after the disease has been confirmed to be healed.$^{4-9}$ In order to decrease this overall period of morbidity experienced by the patient and with the aim of improving clinical and functional outcome, the patient in current study was initially managed with antitubercular therapy followed by a single stage surgical treatment with the aim of obtaining adequate clearance of the disease and regaining the lost function of knee joint. Informed written consent was taken from the patient prior to the procedure.

We report this case study of a patient with tuberculous arthritis of the knee with extensive joint destruction with adequate response to chemotherapy, managed by TKA in single sitting.

\section{CASE REPORT}

\section{History}

A 22-year-old male presented with a 7 months history of pain and progressively increasing swelling in the left knee joint which was drained once at a local health facility. He, however, developed a sinus at the draining site. Based on clinical suspicion and given the incidence of osteoarticular tuberculosis in this part of the world, he was suspected to have tuberculosis of the knee and was started on empirical antitubercular treatment (ATT) 4 months after the onset of symptoms. The usual protocol which is followed for osteoarticular tuberculosis is HRZE for 3 months (where $\mathrm{H}$ is isoniazid given at a dose of $5 \mathrm{mg} / \mathrm{kg}$; $\mathrm{R}$ is rifampicin at $10 \mathrm{mg} / \mathrm{kg}$; $\mathrm{Z}$ is pyrazinamide at $30 \mathrm{mg} / \mathrm{kg}$ and $\mathrm{E}$ is ethambutol at $25 \mathrm{mg} / \mathrm{kg}$ body weight), followed by HRE for 3 months and subsequently HR for 6 months. The sinus healed with ATT but the 
patient continued to experience disabling pain and stiffness in the knee which led him to quit his clerical job and presented with these complaints to our outpatient department.

\section{Physical Examination}

At the time of examination, a scar mark resulting from healed sinus tract over the lateral aspect of knee was seen. The local temperature was comparable to the opposite side and there was no swelling or tenderness. The patient also had a flexion deformity with a painful flexion arc of 30 to $80^{\circ}$. There was, however, no evidence of knee instability.

\section{Laboratory Tests}

The relevant laboratory investigations were: hemoglobin-15.2 gm/dl, total leukocyte count-9,900/ $\mathrm{mm}^{3}$, ESR-16 mm/hr and CRP-1.6 mg/dl. The liver and renal function tests were within normal limits. Of note, the patient denied any constitutional symptoms and his vital parameters were within normal limits. In order to rule out any coexistent pulmonary tuberculosis, a plain chest radiograph was done which was reported as normal.

\section{Imaging}

Plain X-rays of the knee in two planes revealed destructive tricompartmental arthritis with erosion of both femoral and tibial condyles along with periarticular osteopenia (Fig. 1).

\section{Treatment}

The patient was planned for debridement followed by total knee replacement surgery in a single stage and complete preanesthetic checkup was done. A standard medial parapatellar approach was used and a complete synovectomy along with osseous debridement was performed. Soft-tissue releases were carried out to correct the flexion deformity. Total knee replacement was done with a standard posterior stabilized implant (NRG Scorpio, Stryker Orthopaedics) with tibial stem extension (Fig. 2). Patelloplasty was done as in our routine TKA practice and the worn out articular cartilage (Osgood's grade 4) was debrided. The patient was given cephalosporin coverage for 5 days perioperatively. Postoperative period was uneventful, active knee range of motion and isometric quadriceps exercises were started from first postoperative day and the patient was mobilized on the second postoperative day after removal of suction drain. Sutures were removed on the 14 postoperative day. The histopathological analysis of multiple biopsy specimens from the knee confirmed chronic granulomatous synovitis with caseation necrosis suggestive of mycobacterial infection. However, the tissue cultures and special stains for detecting acid fast bacilli were negative. The patient was restarted preoperatively on four drug antitubercular regimen of HRZE for 3 months which was followed by HRE for 3 months and HR for 6 months. Complete course of ATT was given as patient had incomplete course before. Also, the sinus alone had healed which does not imply that disease itself has healed.

\section{Follow-up}

The patient was followed up regularly at monthly intervals for 3 months and at 3 monthly intervals thereafter. One year after TKA, the patient has remained free of systemic and local infection and with a knee range of motion from 0 to $100^{\circ}$ without any clinical or radiological deterioration. Patient's laboratory parameters including the inflammatory markers did not show a rising trend at any point in time. Antitubercular treatment was discontinued

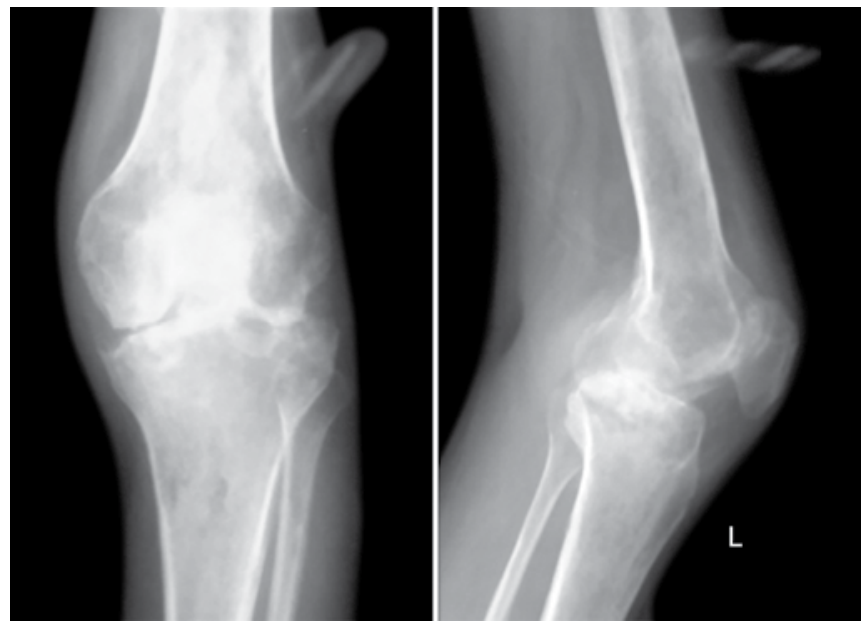

Fig. 1: Preoperative $X$-rays: AP and lateral views showing destructive knee arthritis due to tuberculosis

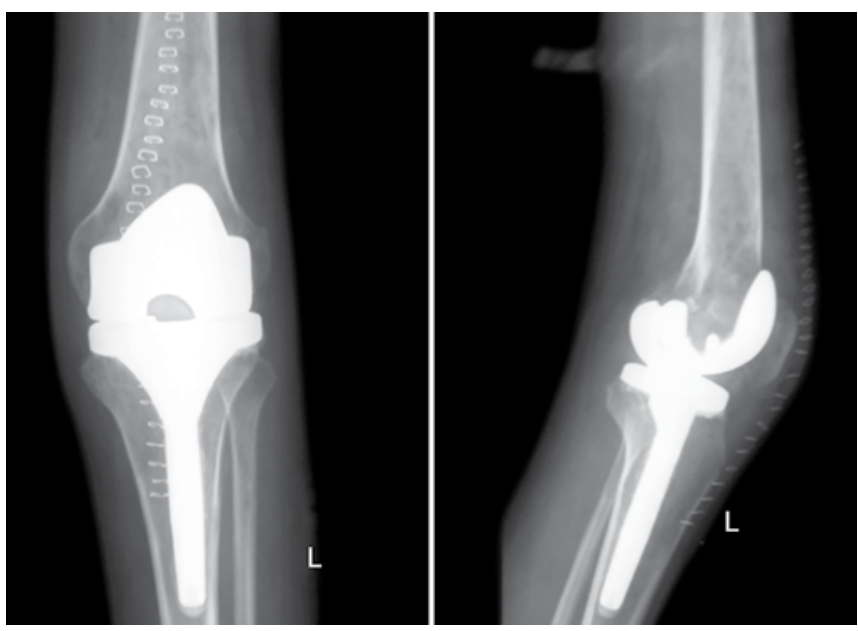

Fig. 2: Postoperative $X$-rays AP and lateral views showing wellfixed and well-positioned prosthesis 
at the end of 1 year. Knee Score (Knee Society) improved from 23 (preoperative) to 76 (postoperative). Functional Score (Knee Society) improved from 45 (preoperative) to 70 (postoperative). Radiographs confirmed a well-fixed and well-positioned stemmed prosthesis and no evidence of recurrent tuberculosis.

\section{DISCUSSION}

Given the prevalence of disease and the morbidity it entails, osteoarticular tuberculosis has long drawn the attention of many workers and it continues to do so. The question of whether a total joint arthroplasty should be attempted in a patient with a current or previous infection of tuberculosis continues to arouse controversy.

Joint replacement in the active stage of disease had long been considered to be a contraindication. ${ }^{10-12}$ However, numerous authors have described total hip arthroplasty in healed tubercular coxitis and the procedure is well accepted these days. ${ }^{13,14}$ Following good results with no reactivation postmetal instrumentation in patients with active spinal tuberculosis and given the in vitro studies which have compared the adherence and biofilm properties of mycobacterium tuberculosis suggesting that the bacterial rarely adheres to a metal surface with little or no biofilm formation, total hip arthroplasty has been done in some cases with advanced active tuberculosis with good functional results and low risk of reactivation. ${ }^{15-18}$ They have recommended that when the infected tissue can be debrided and adequate antitubercular therapy is instituted the outcome of joint arthroplasty may not be adversely affected. Yadav et al reported successful management of periprosthetic tuberculosis in a patient with TKR 14 years after the index surgery with antituberculous drugs only. ${ }^{19}$

Such evidence is however lacking in case of TKA. Some workers have reported the results of TKA in active disease after the diagnosis was initially missed and the disease was diagnosed based on histopathology findings post procedure with a high incidence of reactivation of the disease as these patients did not receive preoperative chemotherapy. Others have recommended a two stage procedure in which the first stage is designed to ensure adequate clearance of the disease and placement of an antibiotic laden cement spacer followed by total knee replacement in the second stage after ensuring quiescent disease. $^{7-9}$

To the best of our knowledge, single stage TKA in active/healing tubercular disease is very uncommonly reported. The difficulties which are encountered in planning such an endeavor includes a risk of reactivation or flare up of the disease requirement of an implant system with maximum flexibility in terms of augmentation, fixation, modularity, and constraint to cover for severe bone loss and damage to the collateral ligaments and significant technical challenge involved in terms of exposure and soft tissue balancing.

In the present case, the diagnosis of knee joint tuberculosis was not confirmed histologically preoperatively as the patient received ATT on the basis of clinical suspicion but disease was under control clinically and by laboratory parameters. Given the good clinical response to antitubercular therapy in terms of decreasing the inflammatory symptoms and achievement of normal laboratory parameters, such an approach could be attempted. Prior to joint replacement the patient was significantly crippled by the disease. Postoperatively the patient reported consistently good outcomes and was able to get back to his occupation and carry on with an unrestricted lifestyle as a result of the procedure. However, given the high risk for reactivation of local and/or systemic disease, prolonged postoperative surveillance will be necessary.

\section{CONCLUSION}

Single stage prosthetic knee joint arthroplasty can be safely performed under adequate ATT coverage in healing stage/treatment phase of the disease provided the response to such a medical treatment is good. Such a procedure provides symptomatic relief, functional improvement and early return to activity. Patient counseling regarding compliance with the antitubercular regimen and postoperative protocol is very important in ensuring success.

\section{REFERENCES}

1. Tuli SM. Tuberculosis of knee joint. In: Tuli SM, editor. Tuberculosis of the skeletal system. 2nd ed. New Delhi, Jaypee Brothers Medical Publishers (P) Ltd, 1997. p. 97-114.

2. Hoffman EB, Allin J, Campbell JA, Leisegang FM. Tuberculosis of the knee. Clin Orthop Relat Res 2002 May;398:100-106.

3. Leclere LE, Sechriest II VF, Holley KG, Tsukayama DT. Tuberculous arthritis of the knee treated with two-stage total knee arthroplasty: a case report. J Bone Joint Surg Am 2009; 91(1):186-191.

4. Bae DK, Yoon KH, Kim HS, Song SJ. Total knee arthroplasty in stiff knees after previous infection. J Bone Joint Surg Br 2005;87-B(3):333-336.

5. Eskola A, Santavirta S, Konttinen YT, Tallroth K, Lindholm ST. Arthroplasty for old tuberculosis of the knee. J Bone Joint Surg Br 1988;70(5):767-769.

6. Besser MI. Total knee replacement in unsuspected tuberculosis of the joint. Br Med J 1980 Jun;280(6229):1434.

7. Wray CC, Roy S. Arthroplasty in tuberculosis of the kneetwo cases of missed diagnosis. Acta Orthop Scand 1987;58(3): 296-298.

8. Su JY, Huang TL, Lin SY. Total knee arthroplasty in tuberculous arthritis. Clin Orthop Relat Res 1996 Feb;323:181-187.

9. Gale DW, Harding ML. Total knee arthroplasty in the presence of active tuberculosis. J Bone Joint Surg Br 1991;73(6):1006-1007. 
10. Babhulkar S, Pande S. Tuberculosis of the hip. Clin Orthop Relat Res 2002 May;398:93-99.

11. Kim YY, Ko CU, Ahn JY, Yoon YS, Kwak BM. Charnley low friction arthroplasty in tuberculosis of the hip: An 8- to 13-year follow-up. J Bone Joint Surg Br 1988;70(5):756-760.

12. Hardinge $\mathrm{K}$, Cleary J, Charnley J. Low-friction arthroplasty for healed septic and tuberculous arthritis. J Bone Joint Surg Br 1979;61(2):144-147.

13. Yoon TR, Rowe SM, Santosa SB, Jung ST, Seon JK. Immediate cementless total hip arthroplasty for the treatment of active tuberculosis. J Arthroplasty 2005;20(7):923-926.

14. Neogi DS, Yadav CS, Kumar A, Khan SA, Rastogi S. Total hip arthroplasty in patients with active tuberculosis of the hip with advanced arthritis. Clin Orthop Relat Res 2010;468(2): 605-612.
15. Ha KY, Chung YG, Ryoo SJ. Adherence and biofilm formation of staphylococcus epidermidis and mycobacterium tuberculosis on various spinal implants. Spine 2005;30(1):38-43.

16. Govender S. The outcome of allografts and anterior instrumentation in spinal tuberculosis. Clin Orthop Relat Res 2002 May;398:60-66.

17. Wang YQ, Wang JS, Xu Z, Li Y, Wang H. Total hip arthroplasty for active tuberculosis of the hip. Int Orthopaed 2010;34(8):1111-1114.

18. Wang Q, Shen H, Jiang $Y$, et al. Cementless total hip arthroplasty for the treatment of advanced tuberculosis of the hip. Orthopedics 2011;34(2):90.

19. Neogi DS, Kumar A, Yadav CS, Singh S. Delayed periprosthetic tuberculosis after total knee replacement: is conservative treatment possible? Acta Orthop Belg 2009;75(1):136-140.

\section{Erratum}

The title of the article "Prevalence of Knee Osteoarthritis and its Correlation in Women of Rural and Urban Parts of Hoshiarpur (Punjab)" [Jan-Mar 2015, Vol. 49(1):32-36] was an error and should be read as "Prevalence of Knee Osteoarthritis and its Correlates in Women of Rural and Urban parts of Hoshiarpur (Punjab)". 